\title{
THE PERFORMANCE OF ENERGY HARVESTED WIRELESS SENSOR NETWORK IN SINGLE-HOP AND MULTI-HOP NETWORK SCENARIO
}

\author{
Vigneswara Rao Gannapathy ${ }^{1}$, Ahamed Fayeez Bin Tuani Ibrahim ${ }^{2}$, Zahriladha Bin Zakaria ${ }^{3}$, \\ Kok Swee Leong ${ }^{4}$ \\ ${ }^{l}$ Lecturer, Department of Electronics and Computer Engineering, Universiti Teknikal Malaysia Melaka, Melaka \\ (UTeM), Malaysia \\ ${ }^{2}$ Lecturer, Department of Electronics and Computer Engineering, Universiti Teknikal Malaysia Melaka, Melaka \\ (UTeM), Malaysia \\ ${ }^{3}$ Lecturer, Department of Electronics and Computer Engineering, Universiti Teknikal Malaysia Melaka, Melaka \\ (UTeM), Malaysia \\ ${ }^{4}$ Lecturer, Department of Electronics and Computer Engineering, Universiti Teknikal Malaysia Melaka, Melaka \\ (UTeM), Malaysia
}

\begin{abstract}
Most of the sensor nodes in Wireless Sensor Network (WSN) are powered by batteries, therefore the lifetime of the sensor nodes are fully relying on its power efficiency. In addition to that, the wireless sensor nodes are commonly placed, distributed and installed in the place where difficult to reach, thus it becomes inconvenient especially when replacing the batteries and leads to high maintenance cost. Due to this reason, batteries and energy source of the sensor nodes has been replaced with more sustainable energy source such as energy harvested source. However, due to some reasons, especially in dense network environment, the energy harvested WSN performs poorly and its network performance is not optimized enough. One of the factors that lead to the low performance of energy harvested WSN is the placement and location of sensor nodes in the network, its network configuration (i.e. single-hop and multi-hops) and the number of sensor nodes used. Therefore, in this study, we investigated and studied the performance of energy harvested WSN in different configuration such as single-hop and multi-hops and in terms of its transmission time (delay), power efficiency, and throughput. The real time energy harvested WSN is deployed for the purpose measurement and analysis.
\end{abstract}

Keywords: Energy Harvested Nodes, Wireless Sensor Network (WSN), Multi-hop

\section{INTRODUCTION}

Nowadays, the wireless sensor network (WSN) is the popular issue all over the world especially for the energy harvested WSNs. The WSN is connecting the sensor nodes wirelessly to form a network [1]. The energy harvested WSN uses harvested energy to power up the wireless sensor nodes.

Since many years ago, the wireless sensor node has been utilized in different fields. The sensor is a device that can detect the changes on a physical condition and produces some measurable results to be transmitted.

However, most of wireless sensor nodes are powered by batteries, in which case the power-efficiency decides the life time of the sensors. On the other hand, the wireless sensor nodes are normally placed in the location which is hard to reach, therefore changing batteries regularly can be inconvenient and most of time will be costly too [6][7]. Hence, batteries have to be replaced with alternative energy sources which are more sustainable and feasible to operate in the environment where the sensors are stationed.

Therefore, the energy harvested WSN that could sustain for years without fully relying on battery source is developed. Among the solutions for that is using a green technologies and harvest unlimited source energy with harvesting solution such as solar energy, wind energy, vibration energy, and thermal energy [8][9][10].

However, the network performance of energy harvested WSNs is not optimized enough. The transmission with wireless sensor nodes produced interferences and noises that 
degrade the performance of the network significantly [11][12]. In addition to that, the packet loss rate is also high in energy harvested WSN due to the network configuration and its characteristics of self powered network [13].

In this work, we investigated the efficiency, reliability and robustness of energy harvested WSNs. Besides, the performance of the network is studied in term of power consumption, latency (response time) and throughput.

\section{NETWORK CONFIGURATION}

The result of this work is divided to two different parts that comprised of single hop and multiple hops. Four parameters such as transmission time, throughput, power consumption and receive signal strength indication (RSSI) are studied and investigated in this work. The constant and manipulate variable that assigned throughout this study are the payload and also the distance respectively. The payload of the network nodes are 50 bytes.

For single hop network scenario, the measurement is taken by transmitting a payload of 50 bytes from the transmitter to the receiver by varying the distance between them. Figure 1 shows the network scenario of single-hop configuration.

In multi-hops WSN, the source node will relay the information to its destination through some intermediates nodes due to transmission range limitation. The Zigbee based energy harvested wireless sensor node used in this work only limited up to 20 meters in transmission range. If source node needs to transmit the information to the destination node that is 100 meters away from each other, then this transmission requires at least four intermediate nodes. Figure 2 shows the network scenario of multi-hop configuration.

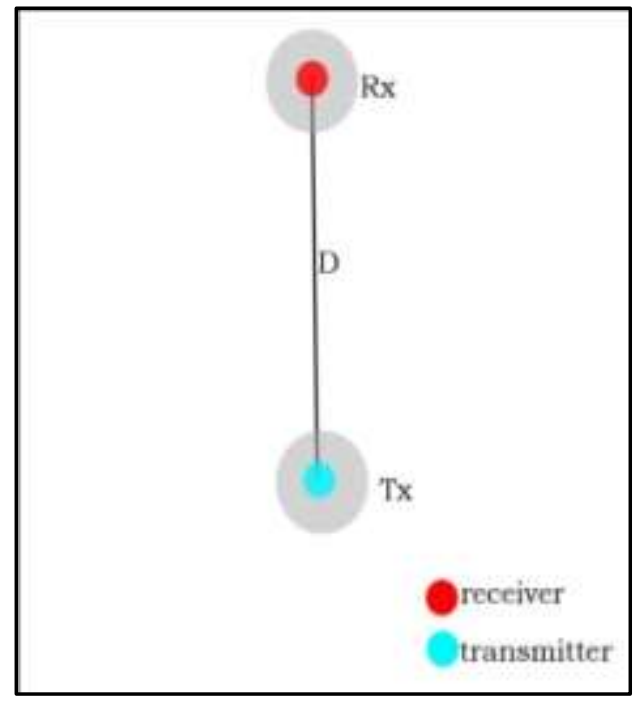

Fig 1: Single-hop Network Scenario

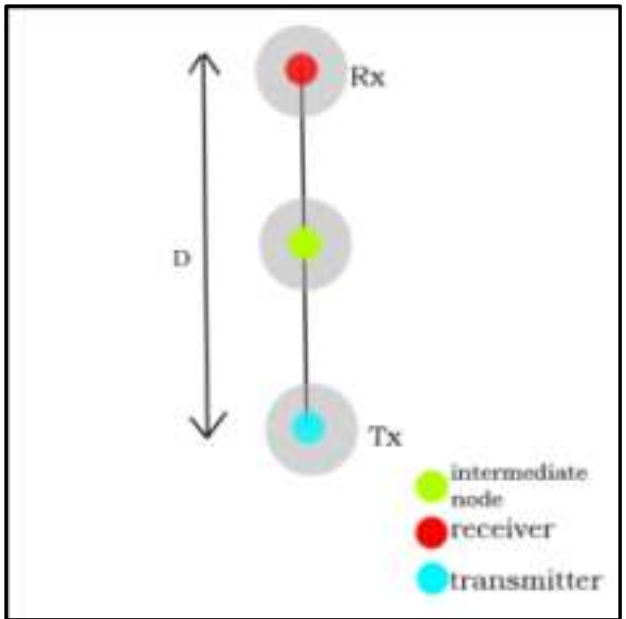

Fig 2: Multi-hop Network Scenario

\section{SINGLE-HOP NETWORK PERFORMANCE}

From the above experiment, we observed that the energy harvested sensor nodes (i.e. zigbee) will only operates to transmit the frame if the distance between the transmitter and receiver is up to 20 meters [3][4][5][6]. For single-hop transmission, when the distance between the transmitters and receivers are increased, thus it increased the frame transmission time accordingly. This is due to the high consumption of power and demands of higher energy in order to transmit a single frame over the long distance. This will subsequently increase the transmission time.

The Received Signal Strength Indicator (RSSI) is used in this study to measure received signal strength in a wireless environment. The RSSI is the measurement of the power level that being received by the receiving node after gone through all the losses from the antenna to the end cables. Therefore, the higher the RSSI indication, the stronger is the signal. When the RSSI is represented in a negative value or it is closer to the value of 0 , thus it has a stronger received signal. The RSSI can be obtained by using the following formula:

RSSI $=10 \log \left[\left(\frac{P_{t} G_{r} G_{t}}{P_{\text {ref }}}\right)\left(\frac{c}{4 \pi f d}\right)^{2}\right]$

Based on the above formula, we can obtain that the RSSI value is proportional to $P t$ (transmission power). With the increase of the distance between the transmitter and receiver, it will increase the transmission power accordingly and therefore it will influence the RSSI value proportionally.

\subsection{Time Response (Transmission Time) in Single- hop Transmission}

Response time is the total amount of time is taken to respond to a request for service. In this work, the response time is measured and recorded by using the digital stopwatch. 
Table 1: The Response Time of Single-hop Transmission

\begin{tabular}{|l|l|}
\hline Transmission Range (meter) & Time (s) \\
\hline 5 & $\mathbf{0 . 9 4 7}$ \\
\hline 10 & $\mathbf{1 . 0 2 3}$ \\
\hline 15 & $\mathbf{4 . 4 2 6}$ \\
\hline 20 & $\mathbf{5 . 8 7 1}$ \\
\hline 25 & - \\
\hline
\end{tabular}

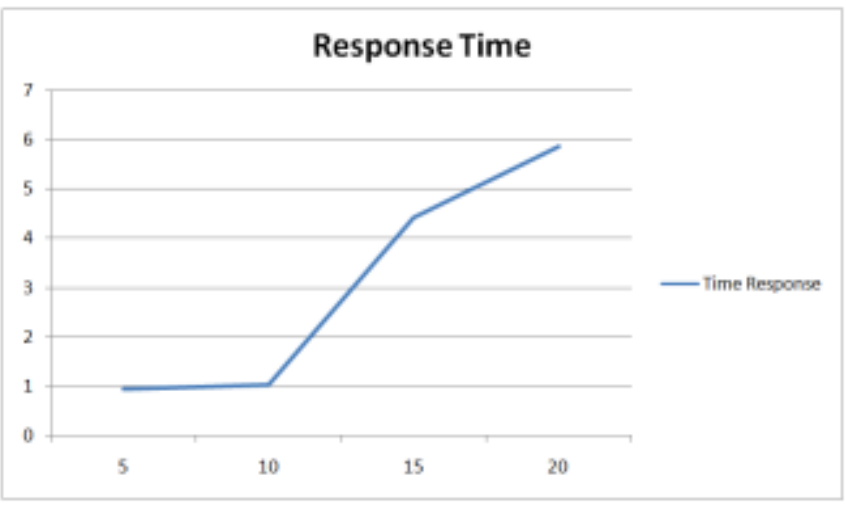

Fig 3: Response Time (s) of Single-hop Transmission over the Distance (Transmission Range), m, of Transmitting and Receiving Nodes

The above table and figure show the relationship between the time response and distance (transmission range). Based on the above result in Figure 3, it clearly can be seen the response time increase accordingly with the increase the distance between the transmitter and receiver.

\subsection{Throughput in Single-hop Transmission}

The throughput is defined as a successful rate of the packet transmission over a communication channel. The packet may be delivered over a physical or logical link, or pass through a certain network node to reach to the end receiver. In reality, the throughput is fundamentally known as bandwidth consumption in which can be compute mathematically by given payload and frame transmission time. The throughput of the single-hop transmission can be obtained by dividing the payload size with the average time required for the packet transmission. The throughput of single-hop transmission is given as follow:

Throughput (bps) $=\frac{8 \times \text { Payload size (Bytes ) }}{\text { Average Frame Transmission Time }}$

Table 2: Throughput of Single Hop

\begin{tabular}{|l|l|}
\hline Transmission Range (meter) & Throughput (bps) \\
\hline 5 & $\mathbf{4 2 2 . 3 9}$ \\
\hline 10 & $\mathbf{3 9 1 . 0 1}$ \\
\hline 15 & $\mathbf{9 0 . 3 8}$ \\
\hline 20 & $\mathbf{6 8 . 1 3}$ \\
\hline 25 & - \\
\hline
\end{tabular}

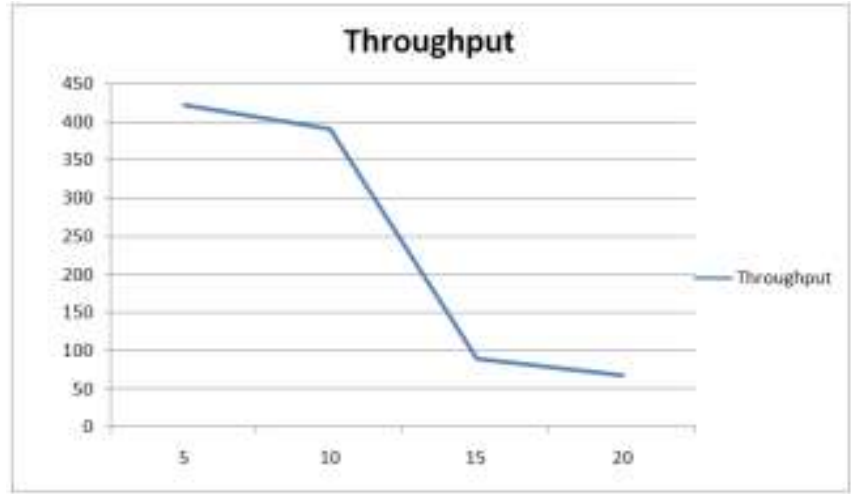

Fig 4: Throughput (bps) of Single-hop Transmission over the Distance (Transmission Range), $\mathrm{m}$.

It is clearly can be observed in Figure 4 that, if the distance between the transmitter and receiver is increased, it consequently increase the frame transmission time thus drop the throughput significantly.

\subsection{Power Consumption in Single-hop \\ Transmission}

In this work, the power consumption (or transmission power), for a node to transmit the entire frame to the receiver, is calculate using the value of voltage $(\mathrm{V})$ and current (I). The voltage (V) and current (I) is measured as per configuration shown in Figure 5 and 6.

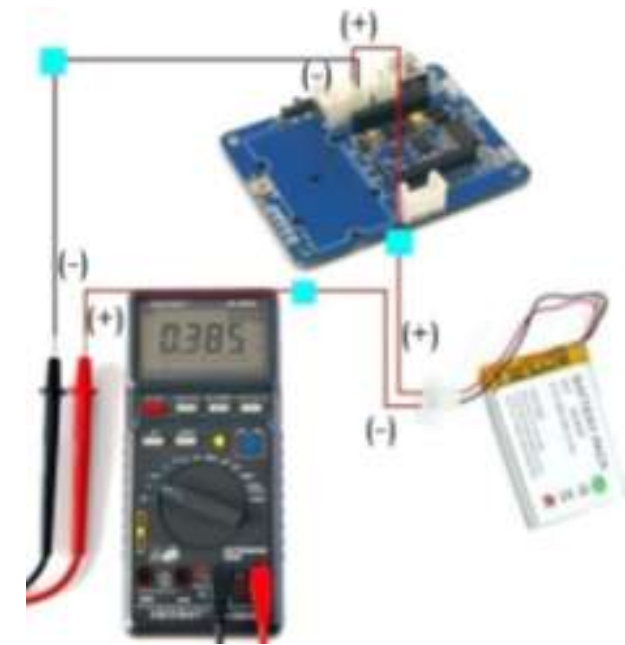

Fig 5: Measurement of Current

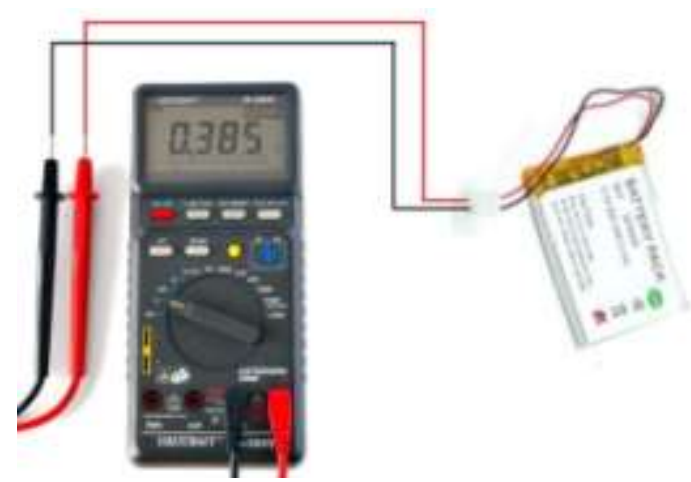

Fig 6: Measurement of Voltage 
Table 3: Power Consumption of Single Hop

\begin{tabular}{|c|c|c|c|}
\hline \multirow{2}{*}{$\begin{array}{l}\text { Transmission } \\
\text { Range } \\
\text { (meter) }\end{array}$} & \multicolumn{3}{|c|}{ Power Consumption } \\
\hline & $\begin{array}{l}\text { Voltage } \\
(V)\end{array}$ & $\begin{array}{l}\text { Current, I } \\
(m A)\end{array}$ & $\begin{array}{l}\text { Power } \\
(m W)\end{array}$ \\
\hline 5 & 3.576 & 0.08 & 0.286 \\
\hline 10 & 3.623 & 5.08 & 18.40 \\
\hline 15 & 3.444 & 15.98 & 55.03 \\
\hline 20 & 3.511 & 22.44 & 78.79 \\
\hline 25 & - & - & - \\
\hline
\end{tabular}

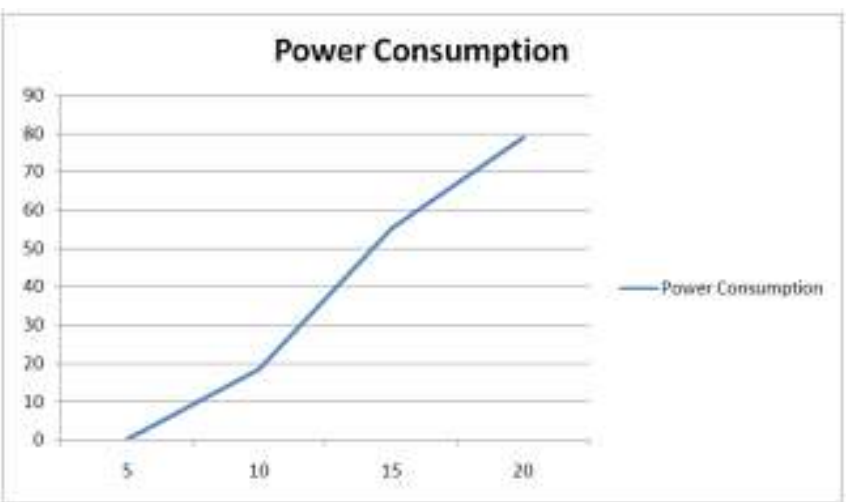

Fig 7: Power Consumption $(m W)$ of Single-hop over the Distance (Transmission Range), $\mathrm{m}$.

Based on the result obtained in Figure 7, it is clearly can be seen that the power consumed to transmit a packet from a node to another node is increased accordingly over the distance. For instant, the power consumed to transmit a packet from the transmitter to the receiver of the distance of 5 meter distance is $0.286 \mathrm{~mW}$, meanwhile 20 meter is $78.79 \mathrm{~mW}$.

\section{MULTI-HOP NETWORK PERFORMANCE}

Due to the limitation between the transmitter and the receiver that can only transmits up to 20 meters in distance, therefore a multi-hop transmission concept used in this study. Multi-hop is a wireless networks architecture that uses two or more intermediate wireless hops to convey or transmit the information from a source to a destination.

This will allow the frame to be transferred from the transmitter to the receiver to the distance which is longer than 20 meters. For instant, four intermediate nodes are required to transmit a frame if the source node is 100 meter away from the destination node.

\subsection{Time Response (Transmission Time) in Multi-}

\section{hop Network Scenario}

The time that required by a node to transmit a packet over multi-hop environment is noticeably longer than time required by the single-hop. This is due to the long distance between the source and destination nodes to transmit a single packet. In other word, when more intermediate nodes are added in the network, it will increase the distance between the source and destination and it subsequently increase the packet transmission time.

This is because the transmitters need to transmit the frame to the receiver that is next to them (intermediate nodes) before it reaching to its final destination. The processing time at each intermediate node will lead to higher time consumption in multi-hop network environment. For instance, the transmitter sends the frame to the intermediate node (receiver) which is next of it; then the receiving node will receive the frame, process it and then pass to the next intermediate node. The process will continue until the packet reaches to its final destination node. This particular process will definitely increase the time as well as lower the throughput of the network. This performance clearly can observed in Figure 8.

Table 4: Time Response in Multiple Hops Network Scenario

\begin{tabular}{|l|l|}
\hline $\begin{array}{l}\text { Transmission Range } \\
\text { (meter) }\end{array}$ & $\begin{array}{l}\text { Time Response } \\
\text { (s) }\end{array}$ \\
\hline $\begin{array}{l}\text { 20 } \\
\text { (without intermediate nodes) }\end{array}$ & 5.871 \\
\hline $\begin{array}{l}40 \\
\text { (with 1 intermediate nodes) }\end{array}$ & $\mathbf{8 . 0 0 1}$ \\
\hline $\begin{array}{l}60 \\
\text { (with } 2 \text { intermediate nodes) }\end{array}$ & 12.439 \\
\hline $\begin{array}{l}80 \\
\text { (with } 3 \text { intermediate nodes) }\end{array}$ & 15.622 \\
\hline $\begin{array}{l}100 \\
\text { (with 4 intermediate nodes) }\end{array}$ & $\mathbf{2 2 . 7 8 5}$ \\
\hline
\end{tabular}

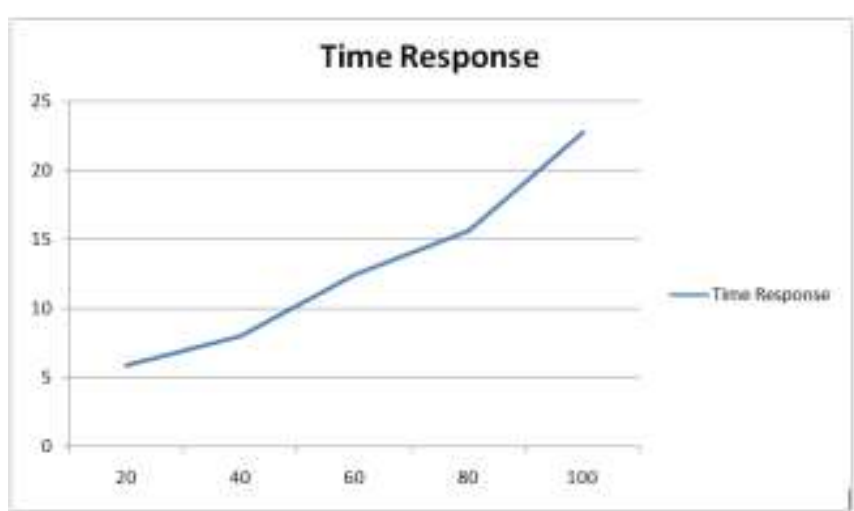

Fig 8: Time Response (s) in Multiple Hops Network Scenario over the Distance (Transmission Range), m, of Transmitting and Receiving Nodes

\subsection{Throughput Achieved in Multi-hop Network}

\section{Scenario}

In multi-hop network environment, throughput can be defined as maximum amount of payload (packet) size that can be transmitted in a time unit. In order to determine the actual throughput, the payload size must be divided with the time taken to transmit the packet from the source node to destination node. Based on our results found in Figure 9, it 
can be obtained that the increase number of intermediates nodes subsequently increase the transmission time (delay) and drop the throughput accordingly.

Table 5: Throughput of Multiple Hops Network Scenario

\begin{tabular}{|l|l|}
\hline $\begin{array}{l}\text { Transmission Range } \\
\text { (meter) }\end{array}$ & $\begin{array}{l}\text { Throughput } \\
\text { (bps) }\end{array}$ \\
\hline $\begin{array}{l}20 \\
\text { (without intermediate nodes) }\end{array}$ & 68.13 \\
\hline $\begin{array}{l}40 \\
\text { (with 1 intermediate nodes) }\end{array}$ & 49.99 \\
\hline $\begin{array}{l}60 \\
\text { (with } 2 \text { intermediate nodes) }\end{array}$ & 32.16 \\
\hline $\begin{array}{l}80 \\
\text { (with } 3 \text { intermediate nodes) }\end{array}$ & 25.60 \\
\hline $\begin{array}{l}100 \\
\text { (with 4 intermediate nodes) }\end{array}$ & 17.56 \\
\hline
\end{tabular}

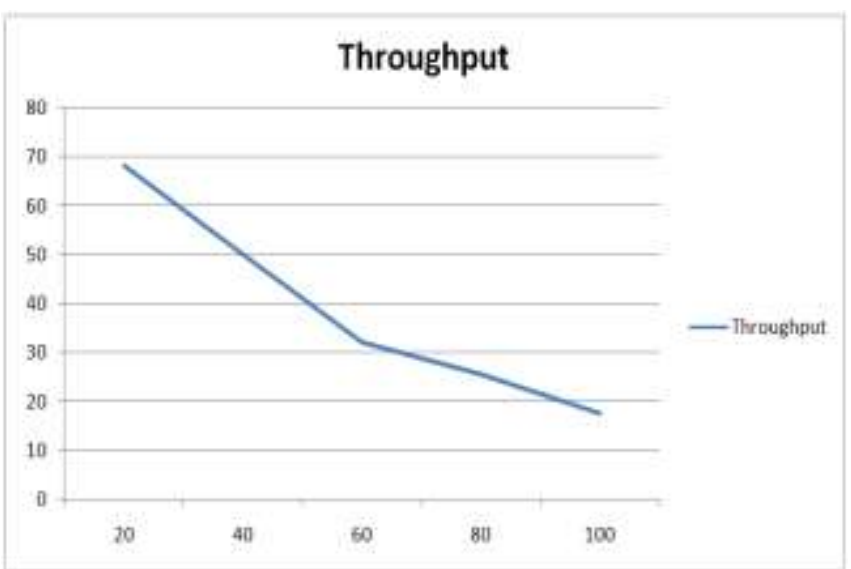

Figure 9: Throughput of Multiple Hops WSN over the Distance (Transmission Range), m.

\subsection{Power Consumption in Multi-hop Network}

\section{Scenario}

In this work, the power consumption (or transmission power), for a node to transmit the entire frame to the receiver, is calculate using the value of voltage $(\mathrm{V})$ and current (I). The voltage (V) and current (I) is measured as per configuration shown in Figure 5 and 6.

Table 6: Power Consumption of Nodes in Multiple hops WSN

\begin{tabular}{|l|l|}
\hline $\begin{array}{l}\text { Transmission Range } \\
\text { (meter) }\end{array}$ & $\begin{array}{l}\text { Total Power } \\
\text { Consumption } \\
\text { (mW) }\end{array}$ \\
\hline $\begin{array}{l}20 \\
\text { (without intermediate nodes) }\end{array}$ & 78.79 \\
\hline $\begin{array}{l}40 \\
\text { (with 1 intermediate nodes) }\end{array}$ & 116.23 \\
\hline $\begin{array}{l}60 \\
\text { (with } 2 \text { intermediate nodes) }\end{array}$ & 176.11 \\
\hline $\begin{array}{l}80 \\
\text { (with } 3 \text { intermediate nodes) }\end{array}$ & 222.91 \\
\hline $\begin{array}{l}100 \\
\text { (with 4 intermediate nodes) }\end{array}$ & 265.44 \\
\hline
\end{tabular}

Based on the result obtained in Figure 10, it is clearly can be seen that the power consumed to transmit a packet from a node to another node is increased accordingly over the distance. For instant, the power consumed to transmit a packet from the transmitter to the receiver of the distance of 20 meter distance is $78.79 \mathrm{~mW}$, meanwhile 100 meter is $265.44 \mathrm{~mW}$.

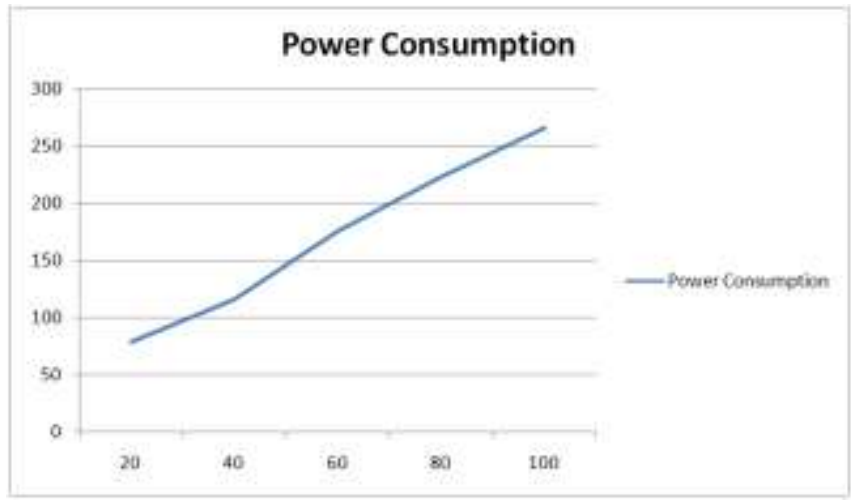

Figure 10: Power Consumption of Multiple hops WSN over the Distance (Transmission Range), $\mathrm{m}$.

\section{CONCLUSION}

In this work, the efficiency, reliability and robustness of energy harvested WSN has investigated. The performance of the network are studied and analyzed in term of power consumption, latency (response time) and throughput. Based on the result, power consumed to transmit a packet from a node to another node is increased accordingly over the distance (transmission range) for both single-hop and multihop configuration. Similar finding is observed for time response (transmission time) and also the throughput. Therefore, it can be concluded that both single-hop and multi-hop configurations are work moderately as well as energy harvested WSN is concern.

\section{ACKNOWLEDGEMENT}

The authors would like to take this opportunity to thanks those who are contributes directly or indirectly in completion of this article and also for their constructive comments. In addition, the authors also would like to express our gratitude to Universiti Teknikal Malaysia Melaka (UTeM) for the support and encouragement.

\section{REFERENCES}

[1] Mumtaz M.Ali AL-Mukhtar, Teeb Hussein Hadi, "Diagnosis of Failures in Zigbee Based Wireless Sensor Networks," IJCSET, Vol 3, Issue 3, 104-108, March 2013.

[2] Alan J Heeger, "Solar Fuels and Artificial Photosynthesis: Science and innovation to change our future energy options," 2012.

[3] D. Gislason, "Zigbee wireless networking", Newest Publications, 2008.

[4] Digi International Inc, "XBee ${ }^{\circledR} / \mathrm{XBee}-\mathrm{PRO}{ }^{\circledR} 2.4$ DigiMesh RF Modules Data Sheet," 2011. 
[5] Behrouz A. Forouzan, "Data Communications and Networking, 4th Edition," pp 7 - 13, 2007.

[6] R. Piyare1, S. Lee, "Performance Analysis of XBee ZB Module Based Wireless Sensor Networks," International Journal of Scientific \& Engineering Research, Volume 4, Issue 4, April-2013.

[7] C. Alippi, "An Adaptive System For Optimal Solar Energy Harvesting In Wireless Sensor Network Nodes," IEEE Transactions on Circuits and SystemsI: Regular Papers, Vol. 55, No. 6, July 2008.

[8] D. Dondi, A. Bertacchini, D. Brunelli, L. Larcher and L. Benini, "Modeling and Optimization of A Solar Energy Harvester System For Self-Powered Wireless Sensor Networks," IEEE Transactions on Industrial Electronics, Vol. 55, No. 7, July 2008.

[9] Manjeshwar and D. P. Agrawal, "TEEN: A Routing Protocol For Enhanced Efficiency In Wireless Sensor Networks" in Center for Distributed and Mobile Computing, ECECS Department, University of Cincinnati, IEEE International Conference.

[10] S. Bandyopadhyay and E. J. Coyle, "An Energy Efficient Hierarchical Clustering Algorithm For Wireless Sensor Networks" in School of Electrical and Computer Engineering, Purdue University, IEEE International Conference.

[11] M. Ye, C. F. Li, G. H. Chen, and J. Wu, "An Energy Efficient Clustering Scheme In Wireless Sensor Networks" in National Laboratory of Novel Software Technology Nanjing University on April 12, 2005, IEEE International Conference.

[12] C. Alippi, R. Camplani, C. Galperti, and M. Roveri, “A Robust, Adaptive, Solar-Powered WSN Framework for Aquatic Environmental Monitoring," IEEE Sensors Journal, Vol. 11, No. 1, January 2011.

[13] Christopher M. Vigorito, Deepak Ganesan, and Andrew G. Barto, "Adaptive Control of Duty Cycling in Energy-Harvesting Wireless Sensor Networks," International Journal of Scientific \& Engineering Research, June 2010.

[14] Vigneswara Rao, Gannapathy., Suaidi, Mohamad Kadim., Johal, Muhammad Syahrir Bin., Chuan, Lim Kim., Ramli, Nordin., Mohamad, Hafizal. (2010). “A Smooth Forwarding Operation in Wireless Mesh Network," in IEEE 10th Malaysia International Conference on Communications (MICC), pp. 83-87.

[15] Vigneswara Rao Gannapathy, Tuani Ibrahim, Ahamed Fayeez, Zahriladha Zakaria, Abdul Rani Othman, Nur Qalbi Jalaudin, "A review on various types of Software Defined Radios (SDRs) in radio communication", IJRET: International Journal of Research in Engineering and Technology eISSN: 2319-1163, pISSN: 2321-7308, Volume: 03 Issue: 12, Dec-2014

[16] Vigneswara Rao Gannapathy, Ahamed Fayeez Bin Tuani Ibrahim, Zahriladha Bin Zakaria, Abdul Rani Bin Othman, Mohamad Kadim Bin Suaidi, “Alleviate Exposed Node Issues In Wireless Mesh Network (WMN) Using A Novel Approach Of Concurrent Medium Access Control (C-MAC) Protocol", IJRET: International Journal of Research in Engineering and Technology eISSN: 2319-1163, pISSN: 2321-7308, Volume: 03 Issue: 11, Dec-2014
[17] Vigneswara Rao Gannapathy, Ahamed Fayeez Bin Tuani Ibrahim, Zahriladha Bin Zakaria, Abdul Rani Bin Othman, Anas Abdul Latiff, "Zigbee-Based Smart Fall Detection And Notification System With Wearable Sensor (e-SAFE)" ", IJRET: International Journal of Research in Engineering and Technology eISSN: 23191163, pISSN: 2321-7308, Volume: 02 Issue: 08, Dec2013

[18] Vigneswara Rao Gannapathy, Ahamed Fayeez Bin Tuani Ibrahim, Zahriladha Bin Zakaria, Abdul Rani Bin Othman, Anas Abdul Latiff, “An Enhancement Of Rts/Cts Control Handshake In CSMA/CA Based Mac Protocol For An Efficient Packet Delivery Over Multihop Wireless Mesh Network (WMN)", IJRET: International Journal of Research in Engineering and Technology eISSN: 2319-1163, pISSN: 2321-7308, Volume: 02 Issue: 10, Dec-2013.

\section{BIOGRAPHIES}

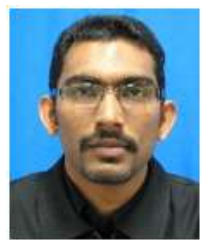

Vigneswara Rao Gannapathy currently serving as a senior lecturer in Universiti Teknikal Malaysia Melaka (UTeM) and he actively involves in research activities which is related to electronics and wireless networking. His research direction has focused on Wireless Mesh Networks which emerged as a key technology for next-generation wireless networking.

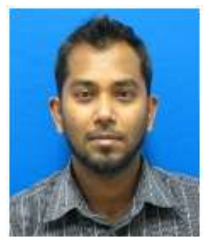

Ahamed Fayeez B Tuani Ibrahim, a Masters holder in Electronics \& Telecommunication, is a vivid computer networking enthusiast. His specific research interest includes low power network design and programming. He is a certified Cisco Network Analyst.

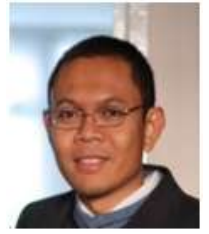

Dr. Zahriladha Zakaria, PhD, MIEEE, BEM, Grad IEM is currently working as a senior lecturer at the Faculty of Electronic and Computer Engineering, University Teknikal Malaysia Melaka. (UTeM). His research interests include a variety of microwave device development such as planar and nonplanar microwave filters, amplifiers and antennas. 